\title{
APPLICATION OF HYBRID AND POLYTOPIC MODELING TO THE STABILITY ANALYSIS OF LINEAR SYSTEMS WITH SATURATING INPUTS
}

\author{
J.M. Gomes da Silva Jr* \\ jmgomes@eletro.ufrgs.br
}

\author{
S. Tarbouriech ${ }^{\dagger}$ \\ tarbourdaas.fr
}

\author{
R. Reginatto* \\ romeudeletro.ufrgs.br \\ ${ }^{*}$ UFRGS - Departamento de Engenharia Elétrica, Av. Osvaldo Aranha 103, 90035-190 Porto Alegre-RS, Brazil. \\ ${ }^{\dagger}$ LAAS-CNRS, 7 Avenue du Colonel Roche, 31077 Toulouse cedex 4, France.
}

\begin{abstract}
This paper is concerned with the problem of stability regions determination for linear systems with saturating inputs. The paper focuses on a critical analysis of two known approaches to model the effect of actuator saturation: hybrid modeling and polytopic modeling. In each case, algorithms to determine ellipsoidal domains of stability for such class of systems are provided in terms of LMIs. The ability of such algorithms in providing large stability domains is analyzed by highlighting the main reasons they incorporate conservativeness, including the influence of the saturation modeling. Two examples are worked out illustrating how significantly the stability domains obtained by such algorithms can differ.
\end{abstract}

KEYWORDS: Control saturation, hybrid modeling, polytopic modeling, stability analysis

\section{RESUMO}

Este artigo trata do problema de determinação de regiões de estabilidade para sistemas lineares com entradas saturantes. O trabalho foca-se em uma análise crítica de duas abordagens conhecidas para o modelamento dos efeitos da saturação de controle: modelamento híbrido e modelamento politópico.

Artigo submetido em $5 / 12 / 2002$

1a. Revisão em 4/08/2003; 2a. Revisão 19/03/2003

Aceito sob recomendação do Ed. Assoc. Prof. José R. C. Piqueira
Em cada caso, algoritmos para determinar domínios de estabilidade para a classe de sistemas considerada são propostos em termos de LMIs. A habilidade de tais algoritmos em fornecer grandes domínios de estabilidade é analisada, enfatizando-se as principais fontes de conservatismo, incluindo o próprio modelamento da saturação. Dois exemplos são apresentados a fim de ilustrar o quão diferente podem ser os domínios de estabilidade obtidos com os diferentes algoritmos.

PALAVRAS-CHAVE: Saturação de controle, modelamento híbrido, modelamento politópico, análise de estabilidade.

\section{INTRODUCTION}

Stability of linear systems with saturating actuators has received a great attention in the recent literature. One important aspect of this class of problems is that the properties of the saturation function give these systems a natural hybrid structure, in the sense that different dynamics can be associated to different operating regions in the state space (Gomes da Silva Jr. e Tarbouriech, 1999b; Gomes da Silva Jr. e Tarbouriech, 1999a). This fact has motivated the use of hybrid systems related tools/techniques in the analysis of these systems. On the other hand, the saturation term in the state space equation can be viewed as a time-varying uncertainty that depends on the state at each instant. Such modeling of the saturation function can be locally incorporated by means of a polytopic system (Molchanov e Pyatnitskii, 1989; Gomes da 
Silva Jr. et al., 1997; Hu e Lin, 2000) which opens another approach, and a corresponding set of results, to deal with the stability analysis and synthesis problems.

One of the most concerning problems in this subject is the determination of asymptotic stability regions for the closedloop system. The motivation for these studies is that, in the presence of control saturation, global stability cannot in general be ensured. Furthermore, when it is possible to compute a global stabilizing control law (see (Sussmann et al., 1994; Burgat e Tarbouriech, 1996)), in general it is difficult to simultaneously guarantee good performance and robustness for the closed-loop system. On the other hand, on the ground of local stabilization, the exact determination of the basin of attraction is possible only in very particular cases. Hence it is important to determine asymptotic stability regions, in order to approximate the basin of attraction (Khalil, 1992).

The proposed methods for generating stability regions for linear systems with saturating inputs explore the special structure of these systems and are mainly based on the concept of Lyapunov domains. Special classes of Lyapunov functions have been considered for such purpose, for instance, piecewise-linear (Gomes da Silva Jr. e Tarbouriech, 1999b), quadratic (see, for example, (Henrion e Tarbouriech, 1999; Gomes da Silva Jr. e Tarbouriech, 1999c; Fong e Hsu, 2000; Hu e Lin, 2000) and references therein) and Lure type (Pittet et al., 1997; Hindi e Boyd, 1998) Lyapunov functions. In order to obtain testable results, the choice of each class of Lyapunov functions is connected to the choice of a convenient representation for the effect of the saturation function. Moreover, both the choice of the Lyapunov function and the saturation modeling are directly related to the conservatism of the results.

In this paper we particularly focus on the hybrid and polytopic representations for linear systems with input saturation and aim at determining ellipsoidal domains of stability. The interest for such domains is mainly motivated by the recent developments concerning numerical algorithms and software packages for solving LMIs and convex optimization problems. This fact allows the implementation of test conditions that can be cast as LMI-based optimization problems where the optimization criteria can be related, directly or indirectly, to the size of the domain of stability to be computed.

Although these methods involve some degree of conservatism, in general the conservatism of the results is not conveniently analyzed or elucidated. It can be noticed a lack of critical comparison between the different approaches. Hence, one of the objectives of this paper is to provide a critical analysis of some methods for computing ellipsoidal regions of asymptotic stability for systems with saturating in- puts. In parallel, we briefly discuss how to use the proposed conditions for the synthesis of local stabilizing control laws. Additionally, we propose two new LMI stability conditions based on a hybrid representation of the saturated system and the use of the S-procedure.

The paper is organized as follows. Section 2 states the problem, related concepts, and definitions. In Sections 3 and 4, two strategies to model the saturation effect are discussed, namely: the modeling by a hybrid system and the modeling by a polytopic system. In each one of these sections the sufficient conditions to be satisfied and the corresponding algorithms for determining the ellipsoidal regions of stability are presented and discussed, including related synthesis issues. Finally two examples are worked out, in Section 5, in order to provide a numerical comparison between the results obtained with the different approaches. The paper is ended with concluding remarks in Section 6.

Notations. For any vector $x \in \Re^{n}, x \succeq 0$ means that all the components of $x$, denoted $x_{(i)}$, are nonnegative. For two vectors $x, y$ of $\Re^{n}$, the notation $x \succeq y$ means that $x_{(i)}-$ $y_{(i)} \geq 0, \forall i=1, \ldots, n . A_{(i)}$ denotes the $i$ th row of matrix $A$. For two symmetric matrices, $A$ and $B, A>B$ means that $A-B$ is positive definite. $A^{\prime}$ denotes the transpose of A. $\operatorname{diag}(x)$ denotes a diagonal matrix obtained from vector x. $1_{m} \triangleq[1 \ldots 1]^{\prime} \in \Re^{m}, 0_{m} \triangleq[0 \ldots 0]^{\prime} \in \Re^{m}$ and $I$ denotes the identity matrix of appropriate dimensions. int $S$ denotes the interior of the set $S$.

\section{PROBLEM STATEMENT}

Consider the continuous-time linear system

$$
\dot{x}(t)=A x(t)+B u(t)
$$

where $x(t) \in \Re^{n}, u(t) \in \Re^{m}, A \in \Re^{n \times n}$ and $B \in \Re^{n \times m}$. Assume system (1) is in closed-loop with the saturated linear control law

$$
u(t)=\operatorname{sat}(K x(t))
$$

where $\operatorname{sat}(\cdot)$ denotes a classical saturation function, i.e. each component $i(i=1, \cdots, m)$ of vector $u(t)$, is defined as follows:

$$
\begin{aligned}
u_{(i)}(t) & =(\operatorname{sat}(K x(t)))_{(i)} \\
& =\left\{\begin{array}{cl}
-\rho_{(i)} & \text { if } K_{(i)} x(t)<-\rho_{(i)} \\
K_{(i)} x(t) & \text { if }-\rho_{(i)} \leq K_{(i)} x(t) \leq \rho_{(i)}(3) \\
\rho_{(i)} & \text { if } K_{(i)} x(t)>\rho_{(i)}
\end{array}\right.
\end{aligned}
$$

where $\rho_{(i)}$ and $-\rho_{(i)}$ represent the control limits.

Due to the saturation term, the closed-loop system is nonlinear:

$$
\dot{x}(t)=A x(t)+B \operatorname{sat}(K x(t))
$$


The polyhedral set

$$
S(K, \rho) \triangleq\left\{x \in \Re^{n} ;\left[\begin{array}{r}
K \\
-K
\end{array}\right] x \preceq\left[\begin{array}{l}
\rho \\
\rho
\end{array}\right]\right\}
$$

is the region of linearity of system (4). Inside this region, the control entries do not saturate and the behavior of the system is described by the linear model

$$
\dot{x}(t)=(A+B K) x(t)
$$

Throughout the paper we assume that the matrix $K$ is such that all the eigenvalues of $(A+B K)$ are placed in the open left half complex plane. In other words, in the absence of control bounds, the closed-loop system would be globally asymptotically stable.

Consider now the ellipsoidal set

$$
\mathcal{E}(P, c)=\left\{x \in \Re^{n} ; x^{\prime} P x \leq c\right\}
$$

where $P=P^{\prime}>0$ and $c>0$.

Definition 2.1 The set $\mathcal{E}(P, c)$ is a region of asymptotic stability for system (4) if: $(i)$ the point $x=0$ is a locally asymptotically stable equilibrium point; $(i i)$ it is contained in the region of attraction of the equilibrium $x=0$.

Definition 2.2 The set $\mathcal{E}(P, c)$ is contractive with respect to system (4) if the function $V(x)=x^{\prime} P x$ is strictly decreasing along the trajectories of (4) in $\mathcal{E}(P, c)-\{0\}$. In particular, if $\mathcal{E}(P, c)$ is contractive, then it is a region of asymptotic stability.

In particular, the problem of determining ellipsoidal regions of stability contained in the region $S(K, \rho)$ is a trivial problem (see (Boyd et al., 1994) for instance). In this paper, we are interested in the study of conditions that allow the determination of stability regions not contained in the region of linearity and, in consequence, that take into account the nonlinear characteristic of the closed-loop system.

\section{HYBRID SYSTEM MODELING}

Due to the specific structure of the saturation function (3), the system (4) naturally exhibits a hybrid structure. This representation consists in dividing the state space in regions called regions of saturation. Inside each region of saturation, the system (4) can be modeled as an affine system or, equivalently, as a system with an additive constant disturbance (Gomes da Silva Jr. e Tarbouriech, 1999b),(Gomes da Silva Jr. e Tarbouriech, 1999a). Thus, the saturated system (4) is viewed, generically, as a hybrid system whose dynamics is piecewise linear (Johansson e Rantzer, 1998).
Consider a vector $\eta(t) \in \Re^{m}$, such that each entry $\eta_{(i)}(t)$, $i=1, \ldots, m$, takes the values 1,0 or -1 in accordance with the saturation function (3) as follows:

$$
\eta_{(i)}(t)=\left\{\begin{array}{cl}
-1 & \text { if } K_{(i)} x(t)<-\rho_{(i)} \\
0 & \text { if }-\rho_{(i)} \leq K_{(i)} x(t) \leq \rho_{(i)} \\
1 & \text { if } K_{(i)} x(t)>\rho_{(i)}
\end{array}\right.
$$

Let $\xi_{j} \in \Re^{m}, j=0,1, \cdots, 3^{m}-1$, represent all possible values of $\eta(t)$. Then, $\forall t, \eta(t)=\xi_{j}$ for some $j$ and hence, each vector $\xi_{j}$ represents a possible combination between saturated and non-saturated control entries. Furthermore, for $\eta(t)=\xi_{j}$, the state vector belongs to a specific region called region of saturation $j$. Each region of saturation is defined by the intersection of half-spaces of the form $K_{(i)} x \leq d_{(i)}$ or $-K_{(i)} x \leq d_{(i)}$, where $d_{(i)}$ can be either $\rho_{(i)}$ or $-\rho_{(i)}$. Generically, the region of saturation associated to $\xi_{j}$ is denoted as:

$$
S\left(R_{j}, d_{j}\right)=\left\{x \in \Re^{n} ; R_{j} x \preceq d_{j}\right\}
$$

where $d_{j} \in \Re^{l_{j}}$ is defined from the entries of $\rho$ and $-\rho$, and $R_{j} \in \Re^{l_{j} \times n}$ is defined from the rows of $K$ and $-K$ (see numerical examples of the regions description in (Gomes da Silva Jr. e Tarbouriech, 1999b) and (Gomes da Silva Jr. e Tarbouriech, 1999a)).

We define $\xi_{0}=0_{m}$ and so the region associated to $j=0$ corresponds to $S(K, \rho)$. In the other regions there is at least one control entry that is saturated. Thus, the motion of the system (4) can be described by the following hybrid system

$$
\begin{aligned}
\dot{x}(t)= & \bar{A}_{j} x(t)+v_{j}, x(t) \in S\left(R_{j}, d_{j}\right), \\
& j=0,1, \cdots, 3^{m}-1
\end{aligned}
$$

with $\bar{A}_{j}=A+B \operatorname{diag}\left(1_{m}-\left|\xi_{j}\right|\right) K$ and $v_{j}=B \operatorname{diag}\left(\xi_{j}\right) \rho$, where $\left|\xi_{j}\right|$ is taken componentwise.

Theorem 1 The function $V(x)=x^{\prime} P x, P=P^{\prime}>0$, is a strictly decreasing Lyapunov function for the saturated system in $\mathcal{E}(P, c)$ if and only if the following conditions hold:

$$
\begin{aligned}
\text { (i) } \quad & x^{\prime} P(A+B K) x+x^{\prime}(A+B K)^{\prime} P x<0, \\
& \forall x \in S(K, \rho) \cap \mathcal{E}(P, c), x \neq 0 \\
\text { (ii) } \quad & x^{\prime} P\left(\bar{A}_{j} x+v_{j}\right)+\left(\bar{A}_{j} x+v_{j}\right)^{\prime} P x<0, \\
& \forall x \in S\left(R_{j}, d_{j}\right) \cap \mathcal{E}(P, c), \forall j, j=1, \ldots, 3^{m}-1, \\
& \text { st } S\left(R_{j}, d_{j}\right) \cap \operatorname{int} \mathcal{E}(P, c) \neq \emptyset
\end{aligned}
$$

Proof: it follows directly from (10). 
Although Theorem 1 provides a necessary and sufficient condition for a set $\mathcal{E}(P, c)$ to be contractive, it still lacks of practical benefit because the conditions (11)- $(i)(i i)$ are not easily solvable with the available numerical methods. In the sequel we present two conditions that, despite being only sufficient for the satisfaction of (11)- $(i)(i i)$, are numerically more tractable.

\subsection{Test Condition 1}

The condition below corresponds to a generalization, to multi-input systems, of the results proposed in (Fong e Hsu, 2000).

Proposition 1 If there exist a matrix $P \in \Re^{n \times n}, P=P^{\prime}>$ 0 , and nonnegative scalars $\gamma_{j}$ and $\tau_{j(i)}, i=1, \ldots, l_{j}$ satisfying the following matrix inequalities

(i) $P(A+B K)+(A+B K)^{\prime} P<0$

(ii) $\left[\begin{array}{cc}P \bar{A}_{j}+\bar{A}_{j}^{\prime} P-\gamma_{j} P & P v_{j}-0.5 R_{j}^{\prime} T_{j}^{\prime} \\ v_{j}^{\prime} P-0.5 T_{j} R_{j} & \gamma_{j} c+T_{j} d_{j}\end{array}\right]<0$

$\forall j, j=1, \ldots, 3^{m}-1$,

such that $S\left(R_{j}, d_{j}\right) \cap \operatorname{int} \mathcal{E}(P, c) \neq \emptyset$

with $T_{j}=\left[\begin{array}{lll}\tau_{j(1)} & \ldots & \tau_{j\left(l_{j}\right)}\end{array}\right]$, then the set $\mathcal{E}(P, c)$ is a region of asymptotic stability for the saturated system (4).

Proof: Relation (12)- $(i)$ implies that relation (11)- $(i)$ is satisfied for all $x \neq 0$. If $x \in S\left(R_{j}, d_{j}\right) \cap \operatorname{int} \mathcal{E}(P, c)$ then $x$ satisfies $\left\{\begin{array}{l}x^{\prime} P x-c \leq 0 \\ R_{j} x-d_{j} \preceq 0\end{array}\right.$. Hence, it follows that a sufficient condition for the satisfaction of (11)-(ii) is that for some nonnegative scalars $\gamma_{j}$ and $\tau_{j(i)}, i=1, \ldots, l_{j}$ one verifies

$$
\begin{array}{r}
x^{\prime}\left(P \bar{A}_{j}+\bar{A}_{j}^{\prime} P\right) x+x^{\prime} P v_{j}+v_{j}^{\prime} P x-\gamma_{j}\left(x^{\prime} P x-c\right) \\
-\sum_{i=1}^{l_{j}} \tau_{j(i)}\left(R_{j(i)} x-d_{j(i)}\right)<0, \forall x, x \neq 0
\end{array}
$$

or, equivalently

$$
\left[\begin{array}{ll}
x^{\prime} & 1
\end{array}\right] G\left[\begin{array}{l}
x \\
1
\end{array}\right]<0 \quad \forall x, x \neq 0
$$

with

$G \triangleq\left[\begin{array}{cc}P \bar{A}_{j}+\bar{A}_{j}^{\prime} P-\gamma_{j} P & P v_{j}-0.5 \sum_{i=1}^{l_{j}} \tau_{j(i)} R_{j(i)}^{\prime} \\ v_{j}^{\prime} P-0.5 \sum_{i=1}^{l_{j}} \tau_{j(i)} R_{j(i)} & \gamma_{j} c+\sum_{i=1}^{l_{j}} \tau_{j(i)} d_{j(i)}\end{array}\right]$

It follows that a sufficient condition for the satisfaction of (14), and, in consequence, for the satisfaction of (11)-(ii) is given by

$$
\left[\begin{array}{cc}
P \bar{A}_{j}+\bar{A}_{j}^{\prime} P-\gamma_{j} P & P v_{j}-0.5 R_{j}^{\prime} T_{j}^{\prime} \\
v_{j}^{\prime} P-0.5 T_{j} R_{j} & \gamma_{j} c+T_{j} d_{j}
\end{array}\right]<0
$$

which completes the proof.

The result of Proposition 1 allows to verify whether a given ellipsoidal set $\mathcal{E}(P, c)$ is contractive or not. It also allows to compute an estimate of the region of attraction of the origin in two different ways:

(a) Given a contractive set $\mathcal{E}(P, c)$ one can try an homothetic expansion by interactively increasing $c$ and testing the condition (15). In this case, the test corresponds to solve an LMI feasibility problem.

(b) Use condition (12) to directly find a contractive set $\mathcal{E}(P, c)$ for system (4). In this case, however, the condition (12)-( $i i)$ becomes a BMI since $P$ and $\gamma_{j}$ will both be decision variables. The solution of a BMI is much more complex than an LMI, and is usually performed by employing some relaxation method (Goh et al., 1996). It is important to remark that, since $P$ is a decision variable, $c$ can be taken as 1 without loss of generality.

A possible relaxation algorithm is as follows.

\section{Algorithm 3.1}

1. Choose $\gamma_{j}=\gamma, \forall j=1, \ldots, 3^{m}-1$.

2. Set $c=1$. Fix $\gamma_{j}, j=1, \ldots, 3^{m}-1$, obtained in the previous step and search for $P$ and $T_{j}$ by optimizing a criterion on the size of $\mathcal{E}(P, c)$ subject to the LMI conditions (12) ${ }^{1}$.

3. Fix $P$ obtained in step 2. Maximize $c$ subject to conditions (12) with $\gamma_{j}$ and $T_{j}, j=1, \ldots, 3^{m}-1$ as free variables ${ }^{2}$.

\section{Go to step 2 .}

The steps 2 and 3 of the algorithm are performed iteratively until a desired precision in the size criterion for $\mathcal{E}(P, c)$ is achieved. Note that, $\left(P, \gamma_{j}, T_{j}\right)$ obtained in step 2 consists in a feasible solution for step 3 with $c=1$. Conversely, $\left(P, c, \gamma_{j}, T_{j}\right)$ obtained in 3 is a feasible solution for step 2 by setting $P$ as $P / c$. Hence the convergence of the algorithm is always ensured.

Remark 1 Computational burden can be reduced in the implementation of inequalities (12) by removing all regions of saturation that are symmetric with respect to the origin, since the satisfaction of (12) in one region of saturation also implies its satisfaction in the region symmetric to it.

\footnotetext{
${ }^{1}$ Since $\gamma_{j}$ are given, the condition becomes an LMI.

${ }^{2}$ This can be accomplished by increasing interactively $c$ and testing (12) as an LMI feasibility problem
} 
Remark 2 The condition (11)-( $i i)$ has been turned into condition (12)-( $i i)$, which can be verified as an LMI test or, in the worse case, as a BMI. In this transformation, however, some conservatism has been introduced due to the following facts:

1. The determination of the stability conditions is based on the S-procedure (see inequality (13)). Indeed, the S-procedure is only a sufficient condition in this case because there is more than a single constraint involved (Boyd et al., 1994).

2. The LMI test (15) implies that

$$
\left[\begin{array}{ll}
x^{\prime} & z^{\prime}
\end{array}\right] \tilde{G}\left[\begin{array}{l}
x \\
z
\end{array}\right]<0
$$

for all $(x, z) \neq 0$, while it would be enough to check the case where $z=1$.

3. The need of a relaxation method implies that we are not certain to find a solution of the problem even if it exists. Moreover, whenever a solution is found, there is no guarantee that solution is the best that could have been found.

4. It is clear that the contractive set $\mathcal{E}(P, c)$ does not necessarily intersect all the regions of saturation. Moreover, only the region that do intersect the set need to be tested. However, if the set $\mathcal{E}(P, c)$ is being synthesized, it is not possible to determine, a priori, whether the searched ellipsoid will intersect or not some of the regions of saturation. In this case, in Algorithm 3.1 the test of (12)-( $i i)$ is performed for all regions of saturation. Hence, it can happen that condition (12)-( $i i)$ is unnecessarily verified in some region $j$.

\subsection{Test Condition 2}

The condition below was mainly inspired by the results presented in (Johansson e Rantzer, 1998) for generic hybrid systems.

Proposition 2 If there exist a matrix $P \in \Re^{n \times n}, P=P^{\prime}>$ 0 , symmetric matrices $M_{j} \in \Re^{l_{j} \times l_{j}}$ with nonnegative entries, and nonnegative scalars $\gamma_{j}$ satisfying the following matrix inequalities

(i) $(A+B K)^{\prime} P+P(A+B K)<0$ (ii) $\left.\begin{array}{cc}P \bar{A}_{j}+\bar{A}_{j}^{\prime} P+R_{j}^{\prime} M_{j} R_{j}-\gamma_{j} P & P v_{j}-R_{j}^{\prime} M_{j} d_{j} \\ v_{j}^{\prime} P-d_{j}^{\prime} M_{j} R_{j} & \gamma_{j} c+d_{j}^{\prime} M_{j} d_{j}\end{array}\right]<0$
$\quad \forall j, j=1, \ldots, 3^{m}-1$,
$\quad$ such that $S\left(R_{j}, d_{j}\right) \cap \mathcal{E}(P, c) \neq \emptyset$

then the set $\mathcal{E}(P, c)$ is a region of stability for the saturated system (4).
Proof: Relation (17)- $(i)$ implies that relation (11)- $(i)$ is satisfied.

Condition (11)-( $i i)$ can be rewritten as:

$$
\begin{gathered}
{\left[x^{\prime} 1\right]\left[\begin{array}{cc}
P & 0 \\
0 & 0
\end{array}\right]\left[\begin{array}{cc}
\bar{A}_{j} & v_{j} \\
0 & 0
\end{array}\right]\left[\begin{array}{l}
x \\
1
\end{array}\right]} \\
+\left[x^{\prime} 1\right]\left[\begin{array}{cc}
\bar{A}_{j}^{\prime} & 0 \\
v_{j}^{\prime} & 0
\end{array}\right]\left[\begin{array}{cc}
P & 0 \\
0 & 0
\end{array}\right]\left[\begin{array}{l}
x \\
1
\end{array}\right]<0
\end{gathered}
$$

$\forall j=1, \ldots, 3^{m}-1, \forall x$ such that $\left\{\begin{array}{l}x^{\prime} P x-c \leq 0 \\ R_{j} x-d_{j} \preceq 0\end{array}\right.$.

Let now $M_{j} \in \Re^{l_{j} \times l_{j}}$ be a symmetric matrix with nonnegative entries and let $\gamma_{j}$ be a nonnegative scalar. It follows that

$$
\begin{array}{r}
{\left[\begin{array}{ll}
x^{\prime} & 1
\end{array}\right]\left[\begin{array}{c}
-R_{j}^{\prime} \\
d_{j}^{\prime}
\end{array}\right] M_{j}\left[\begin{array}{rr}
-R_{j} & d_{j}
\end{array}\right]\left[\begin{array}{c}
x \\
1
\end{array}\right] \geq 0,} \\
\forall x: R_{j} x-d_{j} \preceq 0 \\
\gamma_{j}\left[\begin{array}{ll}
x^{\prime} & 1
\end{array}\right]\left[\begin{array}{cc}
-P & 0 \\
0 & c
\end{array}\right]\left[\begin{array}{c}
x \\
1
\end{array}\right] \geq 0, \forall x: x^{\prime} P x-c \leq 0
\end{array}
$$

Using now the S-procedure, it follows that a sufficient condition for the condition (11)-(ii) is that, for some symmetric matrix $M_{j} \in \Re^{l_{j} \times l_{j}}$ with nonnegative entries and a nonnegative scalar $\gamma_{j}$, one has, $\forall x \neq 0$,

$$
\begin{aligned}
& {\left[\begin{array}{l}
x \\
1
\end{array}\right]^{\prime}\left(\left[\begin{array}{ll}
P & 0 \\
0 & 0
\end{array}\right]\left[\begin{array}{cc}
\bar{A}_{j} & v_{j} \\
0 & 0
\end{array}\right]+\left[\begin{array}{cc}
\bar{A}_{j}^{\prime} & 0 \\
v_{j}^{\prime} & 0
\end{array}\right]\left[\begin{array}{cc}
P & 0 \\
0 & 0
\end{array}\right]\right.} \\
& \left.+\left[\begin{array}{c}
-R_{j}^{\prime} \\
d_{j}^{\prime}
\end{array}\right] M_{j}\left[\begin{array}{c}
-R_{j}^{\prime} \\
d_{j}^{\prime}
\end{array}\right]^{\prime}+\gamma_{j}\left[\begin{array}{cc}
-P & 0 \\
0 & c
\end{array}\right]\right)\left[\begin{array}{c}
x \\
1
\end{array}\right]<0
\end{aligned}
$$

Hence, a sufficient condition for the satisfaction of (21), and, in consequence, for the satisfaction of (11)-(ii) is:

$$
\left[\begin{array}{cc}
P \bar{A}_{j}+\bar{A}_{j}^{\prime} P+R_{j}^{\prime} M_{j} R_{j}-\gamma_{j} P & P v_{j}-R_{j}^{\prime} M_{j} d_{j} \\
v_{j}^{\prime} P-d_{j}^{\prime} M_{j} R_{j} & \gamma_{j} c+d_{j}^{\prime} M_{j} d_{j}
\end{array}\right]<0
$$

which completes the proof.

As it can be concluded from the proofs, Propositions 2 and 1 basically differ in the strategy the S-procedure is handled. In Proposition 2 the constraints are tranformed into quadratic forms (19), (20) before being included in the matrix inequalities. Due to the similarity in the development of the two propositions, all the remarks made about Proposition 1, concerning Algorithm 3.1 and Remarks 1 and 2, apply to Proposition 2 .

Remark 3 In the single input case, since $M_{j}$ is a is a nonnegative scalar it follows that $d_{j}^{\prime} M_{j} d_{j}$ is always nonnegative and so, inequality (22) never admits a feasible solution. In order to avoid this problem we can consider a modified form 
for (19) which yields the following equation as a replacement for (21),

$$
\begin{aligned}
& {\left[\begin{array}{l}
x \\
1
\end{array}\right]^{\prime}\left(\left[\begin{array}{cc}
P & 0 \\
0 & 0
\end{array}\right]\left[\begin{array}{cc}
\bar{A}_{j} & v_{j} \\
0 & 0
\end{array}\right]+\left[\begin{array}{cc}
\bar{A}_{j}^{\prime} & 0 \\
v_{j}^{\prime} & 0
\end{array}\right]\left[\begin{array}{cc}
P & 0 \\
0 & 0
\end{array}\right]\right.} \\
& \left.+\left[\begin{array}{c}
-\bar{R}_{j}^{\prime} \\
\bar{d}_{j}^{\prime}
\end{array}\right] M_{j}\left[\begin{array}{ll}
-\bar{R}_{j} & \bar{d}_{j}
\end{array}\right]+\gamma_{j}\left[\begin{array}{cc}
-P & 0 \\
0 & c
\end{array}\right]\right)\left[\begin{array}{l}
x \\
1
\end{array}\right]<0
\end{aligned}
$$

with $\bar{R}_{j}=\left[\begin{array}{c}R_{j} \\ 0\end{array}\right]$ and $\bar{d}_{j}=\left[\begin{array}{c}d_{j} \\ 1\end{array}\right]$. In this case $M_{j} \in$ $\Re^{2 \times 2}$ and, since $d_{j}$ has always at least one negative element, $\bar{d}_{j}^{\prime} M_{j} \bar{d}_{j}$ can assume negative values depending on the $M_{j}$ entries.

\subsection{Synthesis Issues}

As seen in the previous section, the conditions stated in Propositions 1 and 2 can be directly applied to the problem of estimating the region of attraction of system (4). In this section we discuss how to use these conditions for addressing the following synthesis problem.

Problem 1 Compute a matrix $K$ such that the saturated state feedback control law defined by (3) ensures that for all initial states belonging to a given set of admissible initial conditions $\mathcal{X}_{0}$, the corresponding trajectories of system (4) converge asymptotically to the origin.

In order to address this problem, define diagonal matrices $Q_{1 j}, Q_{2 j}$ and $\Lambda_{j}$ as follows

$$
\begin{gathered}
Q_{1 j(i, i)}=\left\{\begin{array}{rll}
-1 & \text { if } & \xi_{j(i)}=1 \\
0 & \text { if } & \xi_{j(i)}=-1 \\
1 & \text { if } & \xi_{j(i)}=0
\end{array}\right. \\
Q_{2 j(i, i)}=\left\{\begin{array}{rll}
0 & \text { if } & \xi_{j(i)}=1 \\
1 & \text { if } & \xi_{j(i)}=-1 \\
-1 & \text { if } & \xi_{j(i)}=0
\end{array}, \quad i=1, \ldots, m\right.
\end{gathered}
$$

From the definitions (23)-(25), it follows that:

$$
R_{j}=Q_{j} K \triangleq\left[\begin{array}{c}
Q_{1 j} \\
Q_{2 j}
\end{array}\right] K ; \quad d_{j}=\tilde{Q}_{j} \rho \triangleq\left[\begin{array}{c}
Q_{1 j} \\
-Q_{2 j}
\end{array}\right] \rho
$$

For the sake of simplicity, consider $\mathcal{X}_{0}$ as a polyhedral ${ }^{3}$ set described by its vertices:

$$
\mathcal{X}_{0} \triangleq \mathbf{C o}\left\{v_{1}, \ldots, v_{n_{v}}\right\}, \quad v_{s} \in \Re^{n} \forall s=1, \ldots, n_{v}
$$

Based on Proposition 1 the following synthesis result can be stablished.

\footnotetext{
${ }^{3}$ It could be also ellipsoidal or an union of ellipsoidal and polyhedral sets.
}

Proposition 3 If there exist a matrix $W=W^{\prime}>0, W \in$ $\Re^{n \times n}$ a matrix $Y \in \Re^{m \times n}$, matrices $T_{j} \in \Re^{1 \times 2 m}$ with nonnegative entries, and scalars $\gamma_{j}>0, c>0$ and $\beta>1$ such that the following matrix inequalities are satisfied:

$$
\begin{array}{ll}
\text { (i) } \quad & (A W+B Y)+(A W+B Y)^{\prime}<0 \\
\text { (ii) } \quad & {\left[\begin{array}{r}
A W+W A^{\prime}+B \Lambda_{j} Y+Y^{\prime} \Lambda_{j} B^{\prime}-\gamma_{j} W \\
v_{j}^{\prime}-0.5 T_{j} Q_{j} Y \\
v_{j}-0.5 Y^{\prime} Q_{j}^{\prime} T_{j}^{\prime} \\
\gamma_{j} c+T_{j} \tilde{Q}_{j} \rho
\end{array}\right]<0, \forall j, j=1, \ldots, 3^{m}-1} \\
\text { (iii) } \quad\left[\begin{array}{cc}
c & \beta v_{s}^{\prime} \\
\beta v_{s} & W
\end{array}\right] \geq 0, \forall s=1, \ldots, n_{v}
\end{array}
$$

then the gain $K=Y W^{-1}$ solves Problem 1 .

Proof: With $\beta>1$ condition $\left(\right.$ iii) ensures that the set $\mathcal{X}_{0}$ is contained in the ellipsoidal set $\mathcal{E}(P, c)$, with $P=W^{-1}$. Pre and post multiplying $(i)$ and $(i i)$ by the matrix $\left[\begin{array}{cc}P & 0 \\ 0 & I\end{array}\right]$ and considering $K=Y W^{-1}$, it follows, from the proof of Proposition 1, that $\mathcal{E}(P, c)$ is a contractive set for the system (4). Hence if $x(0) \in \mathcal{X}_{0}$, it follows that $x(0) \in \mathcal{E}(P, c)$ which ensures that the corresponding trajectory of system (4) converges asymptotically to the origin.

Considering $W, Y, T_{j}, \gamma$ and $c$ as decision variables, inequalities (27)-(ii) in Proposition 3 are nonlinear and thus very hard to solve. On the other hand, if we fix some of these variables the conditions becomes LMIs. This is the case, for instance, if we fix $\gamma_{j}$ and $T_{j}$. Of course, for given $\gamma_{j}$ and $T_{j}$ it may actually be impossible to find a feasible solution for LMIs (27). In fact, considering a scaling factor $\beta, \beta>0$, the maximum homothetic set to $\mathcal{X}_{0}, \beta \mathcal{X}_{0}$, that can be stabilized considering the fixed $\gamma_{j}$ and $T_{j}$, can be obtained solving the following convex optimization problem with LMI constraints:

$$
\max _{\beta, c, W, Y} \beta
$$

LMIs $(i),(i i),(i i i)$ of Proposition 3

In general we are interested in finding a stabilizing control law that is associated to a domain of stability as large as possible. Of course, fixing variables in (27)-( $i i)$ can lead to very conservative solutions in this sense. In order to overcome this problem we can try to find solutions to the problem using, iteratively, LMI relaxations of conditions (27)-(ii). With this aim we can propose, for example, the following iterative algorithm: 


\section{Algorithm 3.2}

1. Fix $c=1$ and solve (28) without constraints (27)-(ii).

2. Fix $Y$ obtained in the previous step, grid $\gamma\left(\gamma=\gamma_{j}\right.$, $\left.\forall j=1, \ldots, 3^{m}-1\right)$ and find $T_{j}, W$ and $c$ considering the maximization of the scaling factor $\beta$ for each value of $\gamma$ in the grid. Take the solution $W, T_{j}, c$ such that $\beta$ is maximal.

3. Fix $\gamma$ and $T_{j}$, obtained in the previous step, and solve for $W, Y$ and $c$ considering as optimization criteria the maximization of $\beta$.

4. Go to step 2 .

The algorithm stops when no significant improvement in the value of $\beta$ is achieved or when $\beta \geq 1$. For the same arguments given in Algorithm 3.1, the convergence of the algorithm is always ensured. In this case, if the final value of $\beta$ is greater than one, a solution to Problem 1 is given by the final values of $W$ and $Y$.

As pointed out in the analysis case, a drawback of the proposed approach is that conditions (27)-(ii) should be verified in all regions of saturation. It is implicitly assumed that the region $\mathcal{X}_{0}$ will have a non empty intersection with all the regions of saturation, which is not always true. However, since the definition of the regions of saturation depends on the matrix $K$ to be computed, it is impossible to verify this a priori.

The development of synthesis results on the basis of Proposition 2 leads to the following inequality as a replacement for (27)-(ii):

$$
\left[\begin{array}{c}
A_{j} W+W A_{j}^{\prime}+Y^{\prime} Q_{j}^{\prime} M_{j} Q_{j} Y-\gamma_{j} W \\
v_{j}^{\prime}-\rho^{\prime} \tilde{Q}_{j}^{\prime} M_{j} Q_{j} Y \\
v_{j}-Y^{\prime} Q_{j}^{\prime} M_{j} \tilde{Q}_{j} \\
\gamma_{j} c+\rho^{\prime} \tilde{Q}_{j}^{\prime} M_{j} \tilde{Q}_{j} \rho
\end{array}\right]<0
$$

Due to the terms $Y^{\prime} Q_{j}^{\prime} M_{j} Q_{j} Y$, this inequality is more difficult to solve considering $Y$ and $M_{j}$ as decision variables. One way to handle this problem would be to force $M_{j}$ to be positive definite and apply Schur's complement. However, note that this is impossible because, in this case, the term $\gamma_{j} c+\rho^{\prime} \tilde{Q}_{j}^{\prime} M_{j} \tilde{Q}_{j} \rho$ would be always positive. Hence, the application of the test condition 2 for the synthesis problem is not very interesting.

\section{POLYTOPIC SYSTEM MODELING}

Note that the $i$ th entry of the saturated control law defined in (3) can be also written as:

$$
(\operatorname{sat}(K x(t)))_{(i)}=\alpha(x(t))_{(i)} K_{(i)} x(t)
$$

where $0<\alpha(x(t))_{(i)} \leq 1$, is defined as :

$$
\alpha(x(t))_{(i)}=\left\{\begin{array}{cl}
\frac{-\rho_{(i)}}{K_{(i)} x(t)} & \text { if } K_{(i)} x(t)<-\rho_{(i)} \\
1 & \text { if }-\rho_{(i)} \leq K_{(i)} x(t) \leq \rho_{(i)} \\
\frac{\rho_{(i)}}{K_{(i)} x(t)} & \text { if } K_{(i)} x(t)>\rho_{(i)}
\end{array}\right.
$$

The coefficient $\alpha(x(t))_{(i)}$ can be viewed as an indicator of the degree of saturation of the $i$ th entry of the control vector. In fact, the smaller the $\alpha(x(t))_{(i)}$, the farther the state vector is from the region of linearity (5). Notice that $\alpha(x(t))_{(i)}$ is a function of $x(t)$.

Define from the vector $\alpha(x(t)) \in \Re^{m}$ a diagonal matrix $D(\alpha(x(t))) \triangleq \operatorname{diag}(\alpha(x(t)))$. Thus, system (4) can be rewritten as

$$
\dot{x}(t)=(A+B D(\alpha(x(t)) K) x(t)
$$

Theorem 2 The function $V(x)=x^{\prime} P x, P=P^{\prime}>0$, is a strictly decreasing Lyapunov function for the saturated system in $\mathcal{E}(P, c)$ if and only if the following condition hold:

$$
\begin{gathered}
x^{\prime}\left[(A+B D(\alpha(x)) K)^{\prime} P+P(A+B D(\alpha(x)) K)\right] x<0, \\
\forall x \in \mathcal{E}(P, c), x \neq 0
\end{gathered}
$$

Proof: it follows directly from (31).

As the result of Theorem 1, the necessary and sufficient condition given above is hard to test with the available numerical tools. This comes from the fact that $\alpha(x)$ depends on $x$ at each time instant. A way to overcome this difficulty consists in considering, at least locally, a differential inclusion that generates all the trajectories of system (31). In particular, we can consider polytopic differential inclusions. This kind of modeling has been successfully used in the last years for the determination of regions of stability (Gomes da Silva Jr. e Tarbouriech, 1999c; Henrion e Tarbouriech, 1999) as well as for the synthesis of stabilizing control laws in presence of saturating inputs (Gomes da Silva Jr. et al., 1997; Gomes da Silva Jr e Tarbouriech, 2001). Following the same idea of these papers, an interesting alternative modeling was recently proposed in (Hu e Lin, 2000). In the sequel we presented these different approaches of polytopic modeling and the associated sufficient conditions for the satisfaction of (32). 


\subsection{Approach 1}

Let $0<\underline{\alpha}_{(i)} \leq 1$ be a lower bound to $\alpha(x(t))_{(i)}$ and define the vector $\underline{\alpha} \triangleq\left[\underline{\alpha}_{(1)}, \ldots, \underline{\alpha}_{(m)}\right]^{\prime}$. The vector $\underline{\alpha}$ is associated to the following region in the state space:

$$
S\left(K, \rho^{\alpha}\right)=\left\{x \in \Re^{n} ;\left[\begin{array}{c}
K \\
-K
\end{array}\right] x \preceq\left[\begin{array}{l}
\rho^{\alpha} \\
\rho^{\alpha}
\end{array}\right]\right\}
$$

where $\rho_{(i)}^{\alpha} \triangleq \frac{\rho_{(i)}}{\underline{\alpha}_{(i)}}, \forall i=1, \ldots, m$.

Consider now all the possible $m$-order vectors such that the $i$ th entry takes the value 1 or $\underline{\alpha}_{(i)}$. Hence, there exists a total of $2^{m}$ different vectors. By denoting each one of these vectors by $\gamma_{j}, j=1, \ldots, 2^{m}$, define the following matrices: $D_{j}(\underline{\alpha})=D\left(\gamma_{j}\right)=\operatorname{diag}\left(\gamma_{j}\right)$ and $A_{j}=A+B D_{j}(\underline{\alpha}) K$. Note that the matrices $A_{j}$ are the vertices of a convex polytope of matrices. If $x(t) \in S\left(K, \rho^{\alpha}\right)$ it follows that $(A+B D(\alpha(x(t))) K) \in \mathbf{C o}\left\{A_{1}, A_{2}, \ldots, A_{2^{m}}\right\}$. Hence, if $x(t) \in S\left(K, \rho^{\alpha}\right), \dot{x}(t)$ can be determined from an appropriate convex linear combination of matrices $A_{j}$ at time $t$, that is:

$$
\dot{x}(t)=\sum_{j=1}^{2^{m}} \lambda_{j}(x(t)) A_{j} x(t)
$$

with $\sum_{j=1}^{2^{m}} \lambda_{j}(x(t))=1, \quad \lambda_{j}(x(t)) \geq 0$.

It should be pointed out that model (34) represents the saturated system only in $S\left(K, \rho^{\alpha}\right)$. Actually, if $x(t) \in S\left(K, \rho^{\alpha}\right)$, the polytopic model (34) can be used to determine $\dot{x}(t)$.

Proposition 4 If there exist a matrix $P=P^{\prime}>0, P \in$ $\Re^{n \times n}$, a vector $\underline{\alpha} \in \Re^{m}$ and a positive scalar $c$ satisfying the following conditions

$$
\begin{aligned}
& P\left(A+B D_{j}(\underline{\alpha}) K\right)+\left(A+B D_{j}(\underline{\alpha}) K\right)^{\prime} P<0, \\
& \forall j=1, \ldots, 2^{m} \\
& {\left[\begin{array}{cc}
P & \underline{\alpha}_{(i)} K_{(i)}^{\prime} \\
\underline{\alpha}_{(i)} K_{(i)} & \rho_{(i)}^{2} / c
\end{array}\right] \geq 0 \quad \forall i=1, \ldots, m}
\end{aligned}
$$

(iii) $0<\underline{\alpha}_{(i)} \leq 1 \forall i=1, \ldots, m$

then the set $\mathcal{E}(P, c)$ is a region of stability for the saturated system (4).

Proof: Provided conditions (ii) and (iii) are verified it follows that $\mathcal{E}(P, c)$ is contained in $S\left(K, \rho^{\alpha}\right)$ (Gomes da Silva Jr. et al., 1997; Boyd et al., 1994).

Hence, for all $x(t) \in \mathcal{E}(P, c), \forall t \geq 0$, it follows that there exists $\lambda_{j}(x(t))$, with $\sum_{j=1}^{2^{m}} \lambda_{j}(x(t))=1, \quad \lambda_{j}(x(t)) \geq 0$, such that:

$$
\dot{x}(t)=\left(A+B D(\alpha(x(t)) K) x(t)=\sum_{j=1}^{2^{m}} \lambda_{j}(x(t)) A_{j} x(t)\right.
$$

From condition $(i)$ it follows that

$$
\begin{array}{r}
x(t)^{\prime} P \sum_{j=1}^{2^{m}} \lambda_{j}(x(t))\left(A+B D_{j}(\underline{\alpha}) K\right) x(t) \\
+\sum_{j=1}^{2^{m}} x(t)^{\prime} \lambda_{j}(x(t))\left(A+B D_{j}(\underline{\alpha}) K\right)^{\prime} P x(t)<0
\end{array}
$$

or equivalently,

$$
\begin{array}{r}
x(t)^{\prime}(A+B D(\alpha(x(t))) K)^{\prime} P x(t) \\
+x(t)^{\prime} P(A+B D(\alpha(x(t))) K) x(t)<0
\end{array}
$$

Since this reasoning is valid $\forall x(t) \in \mathcal{E}(P, c), x \neq 0, \forall t \geq 0$, from Theorem 2 it follows that $\mathcal{E}(P, c)$ is a region of stability for the saturated system.

Similarly to Propositions 1 and 2, the sufficient condition stated in Proposition 4 allows both to test if a given $\mathcal{E}(P, c)$ is contractive and to determine a contractive set based on some geometric criterion:

- In the first case, since $P$ and $c$ are given, conditions (35)- $(i)(i i)(i i i)$ can be easily tested as an LMI feasibility problem in variable $\underline{\alpha}$.

- In the second case, $P$ and $\underline{\alpha}$ appear as problem variables $^{4}$ and conditions (35)- $(i)$ become BMIs whereas (35)-(ii) (iii) are LMIs. As pointed out in section 3.1, the presence of a BMI constraint makes difficult the direct solution of an optimization problem. A possible relaxation scheme in this case is as follows (see (Gomes da Silva Jr. e Tarbouriech, 1999c) and (Henrion e Tarbouriech, 1999) for more details):

\section{Algorithm 4.1}

\section{Choose $\underline{\alpha}$.}

2. Set $c=1$. Fix $\alpha$ obtained in the previous step, search for $P$ by optimizing a criterion on the size of $\mathcal{E}(P, c)$ subject to the LMI constraints given by (35)- $(i)(i i)$

3. Fix $P$ obtained in step 2 . Minimize $\mu=\frac{1}{c}$ subject to LMI constraints given by (35)- $(i)($ ii $)($ iii $)$ with $\underline{\alpha}$ as free variable.

\footnotetext{
${ }^{4}$ Note that here we can consider without loss of generality $c=1$.
} 


\section{Go to step 2.}

The convergence of Algorithm 4.1 can be concluded by a reasoning similar to Algorithm 3.1.

Remark 4 The conservatism of the condition given by Proposition 4 is due to the modeling of the behavior of the saturated system by a differential inclusion. In fact, (35)- $(i)$ is a necessary and sufficient condition for the quadratic stability of the polytopic system

$$
\dot{x}(t)=\sum_{j=1}^{2^{m}} \lambda_{j}(t) A_{j} x(t)
$$

$\forall \lambda_{j}(t)$ such that $\sum_{j=1}^{2^{m}} \lambda_{j}(t)=1, \quad \lambda_{j}(t) \geq 0$. Note, however, that all trajectories of the saturated system (4) are also trajectories of system (39), but the converse is not necessarily true.

\subsection{Approach 2}

Consider a matrix $\bar{H} \in \Re^{m \times n}$ and define

$$
S(\bar{H}, \rho) \triangleq\left\{x \in \Re^{n} ;\left[\begin{array}{c}
\bar{H} \\
-\bar{H}
\end{array}\right] x \preceq\left[\begin{array}{l}
\rho \\
\rho
\end{array}\right]\right\}
$$

Let now matrices $\Theta_{j}, j=1, \ldots, 2^{m}$ be diagonal matrices whose diagonal elements are equal to 1 or 0 . From these definitions, if $x(t)$ belongs to the set $S(\bar{H}, \rho)$, it can be shown (by convexity arguments) that $\dot{x}(t)$ can be computed by the following polytopic model:

$$
\dot{x}(t)=\sum_{j=1}^{2^{m}} \lambda_{j}(x(t))\left(A+B\left(\Theta_{j} K+\left(I-\Theta_{j}\right) \bar{H}\right) x(t)\right.
$$

with $\sum_{j=1}^{2^{m}} \lambda_{j}(x(t))=1, \quad \lambda_{j}(x(t)) \geq 0$. Similar to the previous section, the model (34) represents the saturated system only in the region $S(\bar{H}, \rho)$.

Proposition 5 (Hu e Lin, 2000) If there exist matrices $P=$ $P^{\prime}>0, P \in \Re^{n \times n}$ and $H \in \Re^{m \times n}$, and a positive scalar $c$ satisfying the following conditions

$$
\begin{aligned}
& \text { (i) } \quad P\left[A+B\left(\Theta_{j} K+\left(I-\Theta_{j}\right) \bar{H}\right)\right] \\
&+\left[A+B\left(\Theta_{j} K+\left(I-\Theta_{j}\right) \bar{H}\right)\right]^{\prime} P<0, \\
& \forall j=1, \ldots, 2^{m} \\
& \text { (ii) } \quad\left[\begin{array}{cc}
P & \bar{H}_{(i)}^{\prime} \\
\bar{H}_{(i)} & \rho_{(i)}^{2} / c
\end{array}\right] \geq 0, \forall i=1, \ldots, m
\end{aligned}
$$

then the set $\mathcal{E}(P, c)$ is a region of stability for the saturated system (4).
Proof: Considering that $(i i)$ ensures that $\mathcal{E}(P, c) \subset S(\bar{H}, \rho)$, the proof follows similarly to the one of Proposition 4.

By setting $W=P^{-1}, \gamma=\frac{1}{c}$ one can state the following corollary.

Corollary 3 If there exist a symmetric positive definite matrix $W$, a positive scalar $\gamma$ and a matrix $G$ satisfying the following LMIs:

(i) $\quad A W+B\left(\Theta_{j} K W+\left(I-\Theta_{j}\right) G\right)+W A^{\prime}$ $+\left(\Theta_{j} K W+\left(I-\Theta_{j}\right) G\right)^{\prime} B^{\prime}<0, \forall j=1, \ldots, 2^{m}$

(ii) $\left[\begin{array}{cc}W & G_{(i)}^{\prime} \\ G_{(i)} & \gamma \rho_{(i)}^{2}\end{array}\right] \geq 0 \forall i=1, \ldots, m$

then $\bar{H}=G W^{-1}$ and the set $\mathcal{E}\left(P, \gamma^{-1}\right) \subset S(\bar{H}, \rho)$ is a region of stability for the saturated system (4).

Considering a criterion on the size of the region $\mathcal{E}\left(P, \gamma^{-1}\right)$, the conditions given in Proposition 5 or in Corollary 3 can be straightforwardly applied to the determination of ellipsoidal regions of stability for the saturated system. The main advantage of this approach is that the conditions are LMIs and no relaxation scheme is needed. Note that in this case we can consider $\gamma=1$ without loss of generality. Regarding the conservativity of the modeling, similar comments stated in Remark 4 apply to this approach.

\subsection{Synthesis Issues}

Following the same reasoning presented in section 3.3, the condition proposed in Proposition 4 can be used for synthesis purposes. This result is formalized as follows:

Proposition 6 (Gomes da Silva Jr. et al., 1997) If there exist matrices $W=W^{\prime}>0, W \in \Re^{n \times n}$ and $Y \in \Re^{m \times n}$ and a vector $\underline{\alpha} \in \Re^{m}$, satisfying the following matrix inequalities:

(i) $W A^{\prime}+A W+B D_{j}(\underline{\alpha}) Y+Y^{\prime} D_{j}(\underline{\alpha}) B^{\prime}<0$ $\forall j=1, \ldots, 2^{m}$

(iv) $0<\underline{\alpha}_{(i)} \leq 1, i=1, \ldots, m$

then $K \triangleq Y W^{-1}$ solves Problem 1 .

As in the Proposition 3, inequalities (43)-(i) and (43)-(ii) are nonlinear if we consider $\underline{\alpha}$ and $Y$ as decision variables. 
The same discussion done in the previous section is valid here. A similar iterative algorithm can be proposed in order to find a solution for Problem 1 considering a given set $\mathcal{X}_{0}$ of admissible initial conditions (see (Gomes da Silva Jr e Tarbouriech, 2001)). Note that here the algorithm will be simpler than the Algorithm proposed in section 3.3 since it suffices to iterate two steps: in the first step we fix $\underline{\alpha}$ and in the second we fix $Y$.

On the other hand, the result of Corollary 3 can be used for synthesis purposes by substituting (43)-( $i$ ) and (43)-(ii) by

$$
\begin{aligned}
\text { (i) } \quad & A W+B\left(\Theta_{j} Y+\left(I-\Theta_{j}\right) G\right)+W A^{\prime} \\
& +\left(\Theta_{j} Y+\left(I-\Theta_{j}\right) G\right)^{\prime} B^{\prime}<0, \forall j=1, \ldots, 2^{m} \\
\text { (ii) } \quad & {\left[\begin{array}{cc}
W & G_{(i)}^{\prime} \\
G_{(i)} & \rho_{(i)}^{2}
\end{array}\right] \geq 0 \forall i=1, \ldots, m }
\end{aligned}
$$

In this last case, as in the analysis case, the constraints appear directly as LMIs in the decision variables $W, Y$ and $G$ which avoid the use of relaxation schemes.

\section{NUMERICAL EXAMPLES}

The algorithms to synthesize ellipsoidal stability domains for linear systems with saturation described in the paper will now be applied to two different systems. The goal is to compare the effectiveness of the algorithms in synthesizing large stability domains and to verify the actual effect of the conservative steps involved in each algorithm.

For each system we solve the problem of finding an ellipsoidal asymptotic stability domain by applying each of the methods described in the paper. For each method, we search for the best possible ellipsoid, in the attempt to finding the largest possible region of stability. All the results are plotted to allow a visual comparison of the size of the stability regions obtained with each method.

Example 5.1 We consider first a single input, second order linear system in closed-loop by a linear state feedback with saturation. The parameter of this system are:

$$
\begin{aligned}
& A=\left[\begin{array}{cc}
0.5 & -1 \\
1 & 0.5
\end{array}\right] ; \quad B=\left[\begin{array}{c}
0.5 \\
1
\end{array}\right] ; \\
& K=\left[\begin{array}{ll}
0.278 & -2.139
\end{array}\right] ; \rho=4
\end{aligned}
$$

- Result obtained with the hybrid modeling 1 st condition: The criterion considered was the maximization of the minor axis of the ellipsoidal region (i.e. minimization of the greater eigenvalue of $P$ ). The optimal value of

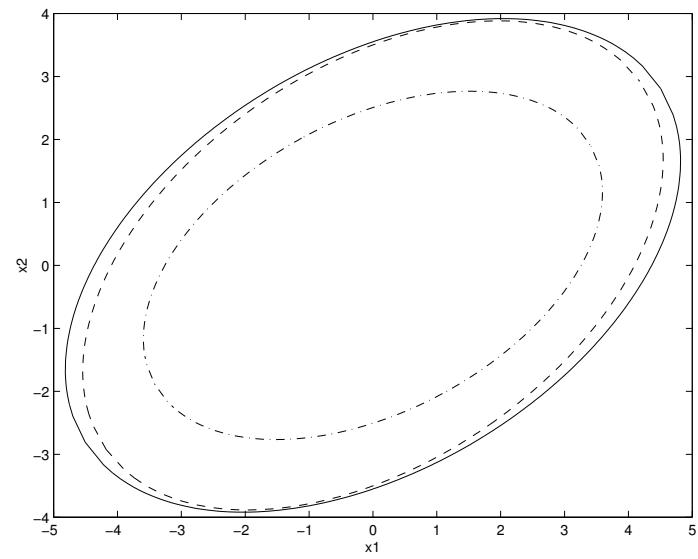

Figure 1: hybrid modeling 1st and 2nd condition (dashdotted); polytopic 1st approach (dashed); polytopic 2nd approach (solid)

this criterion is obtained for $\gamma=2.1$ with

$$
P=\left[\begin{array}{cc}
0.0942 & -0.0517 \\
-0.0517 & 0.1591
\end{array}\right] ; c=1
$$

- Result obtained with the hybrid modeling 2nd condition:

The optimal value of this criterion is obtained for $\gamma=$ 2.1 with

$$
P=\left[\begin{array}{cc}
0.0942 & -0.0517 \\
-0.0517 & 0.1591
\end{array}\right] ; c=1
$$

- Result obtained with the polytopic modeling 1st approach:

Applying the Algorithm 4.1, considering in step 2 the minimization of the greater eigenvalue of $P$ and starting with $\underline{\alpha}=1$ we obtain

$$
P=\left[\begin{array}{cc}
0.0596 & -0.0302 \\
-0.0302 & 0.0816
\end{array}\right] ; c=1
$$

- Result obtained with the polytopic modeling 2nd approach:

One obtains:

$$
P=\left[\begin{array}{cc}
0.0525 & -0.0273 \\
-0.0273 & 0.0793
\end{array}\right] ; c=1
$$

Figure 1 depicts the ellipsoids obtained with the different approaches.

In this case, the regions obtained with the hybrid system modeling approaches are practically identical but smaller than the ones obtained from the polytopic models. 
Example 5.2 Consider now the following multi-input second order linear system with:

$$
\begin{gathered}
A=\left[\begin{array}{cc}
0.1 & -0.1 \\
0.1 & -3
\end{array}\right] ; B=\left[\begin{array}{ll}
5 & 0 \\
0 & 1
\end{array}\right] ; \\
K=\left[\begin{array}{cc}
-0.7283 & -0.0338 \\
-0.0135 & -1.3583
\end{array}\right] ; \rho=\left[\begin{array}{l}
5 \\
2
\end{array}\right]
\end{gathered}
$$

- Result obtained with the hybrid modeling 1 st condition:

Applying the Algorithm 3.1, considering as criterion the maximization of the minor axis of the ellipsoidal region (i.e. minimization of the greater eigenvalue of $P$ ), the best value for $\gamma$ in the first step is 0.25 . With this value one obtains

$$
P=10^{-3}\left[\begin{array}{ll}
0.5886 & 0.0023 \\
0.0023 & 0.2800
\end{array}\right] ; c=1
$$

- No solution was found with the hybrid modeling 2nd condition.

- Result obtained with the 1 st polytopic modeling approach:

Applying the Algorithm 4.1, considering also the maximization of the minor axis of the ellipsoidal region and initializing $\underline{\alpha}$ as $1_{m}$, we obtain

$$
P=10^{-4}\left[\begin{array}{ll}
0.1608 & 0.0001 \\
0.0001 & 0.1592
\end{array}\right] ; c=1
$$

with $\underline{\alpha}=\left[\begin{array}{ll}0.0275 & 0.0034\end{array}\right]^{\prime}$.

- Result obtained with the 2nd polytopic modeling approach:

$$
P=10^{-4}\left[\begin{array}{cc}
0.1542 & -0.0038 \\
-0.0038 & 0.0078
\end{array}\right] ; c=1
$$

Figure 2 depicts the ellipsoids obtained with the different approaches.

Note that the set obtained with the hybrid modeling 1st condition is significantly smaller than the set obtained with the polytopic approaches. Moreover the 2nd polytopic approach gives a less conservative domain than the 1 st one.

\section{CONCLUDING REMARKS}

We have considered the local stability and stabilization problems for saturated linear systems in a comparative study context. We focused on the determination of ellipsoidal domains of stability by employing quadratic Lyapunov functions. This has been done for two different approaches to

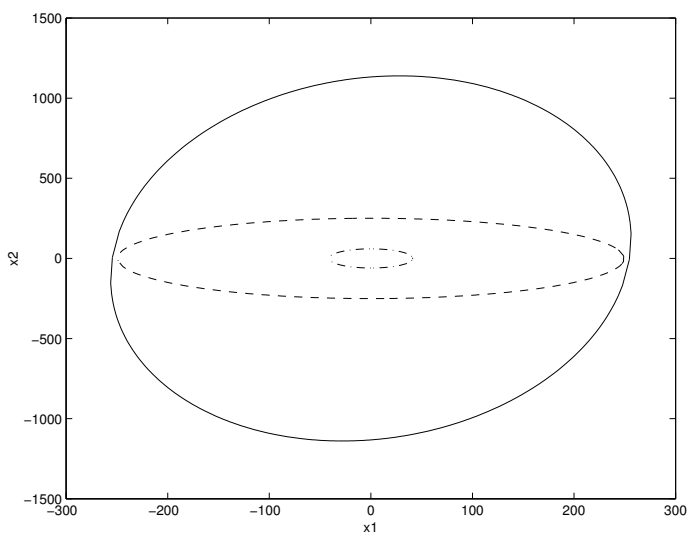

Figure 2: hybrid modeling 1st condition (dash-dotted); polytopic 1st approach (dashed); polytopic 2nd approach (solid)

model the saturated system: a hybrid modeling and a polytopic modeling. Based on the results presented and the simulations provided, it is possible to draw de following conclusions:

- Two main sources of conservativeness can be identified: the modeling and the strategy to develop a testable condition. It is clear that these two aspects are connected, since the modeling determines, in a large extent, the tools that can be applied to obtain testable conditions.

- The main source of conservativeness in the hybrid modeling case comes from the transformation of the problem into a set of matrix inequalities, which requires the use of the S-procedure among other key assumptions.

- The main source of conservativeness in the polytopic modeling can be identified as the abstraction of the modeling. The test condition considers $\lambda_{j}$ as arbitrary functions of time that live between given limits, thus neglecting their intrinsic link to the state.

The synthesis of linear feedbacks is also possible with both modeling strategies. However, the conditions involved with the polytopic modeling are simpler to handle. The difficulties to deal with the synthesis problem with the hybrid modeling come from the fact that the partition of the state space is performed in accordance with the saturation function and, thus, depends on the state feedback gain being designed. As a result, the problem is highly nonlinear and more elaborate relaxations methods have to be employed to reduce the problem to LMIs. This procedure, however, is certainly another source of conservativeness. On the other hand, in the polytopic case, one of the approaches allows to state the test conditions for both analysis and synthesis problems, directly as an LMI, thus avoiding the need for relaxation methods. 
As far as the computational burden is concerned, the two test conditions derived from the hybrid modeling are similar. In this case, a set of $3^{m}$ LMIs have to be solved. On the other hand, the polytopic modeling involves $2^{m}$ LMIs, thus being less computationally expensive.

\section{REFERENCES}

Boyd, S., El Ghaoui, L., Feron, E. e Balakrishnan, V. (1994). Linear Matrix Inequalities in System and Control Theory, SIAM Studies in Applied Mathematics.

Burgat, C. e Tarbouriech, S. (1996). Stability and control of saturated linear systems, in A. Fossard e D. NormandCyrot (eds), Non-Linear Systems, Vol. 2, Chapman \& Hall.

Fong, I.-K. e Hsu, C.-C. (2000). State feedback stabilization of single input systems through actuators with saturation and deadzone characteristics, Proc. of 39st IEEE Conference on Decision and Control (CDC'00), Sydney, Australia.

Goh, K., Safonov, M. G. e Ly, J. H. (1996). Robust synthesis via bilinear matrix inequalities, Int. J. of Robust and Nonlinear Control 6: 1079-1095.

Gomes da Silva Jr., J. M., Fischman, A., Tarbouriech, S., Dion, J. M. e Dugard, L. (1997). Synthesis od state feedback for linear systems subject to control saturation by an lmi-based approach, Proc. of the 2nd IFAC Workshop on Robust Control Design (ROCOND'97), Budapest, Hungary, pp. 229-234.

Gomes da Silva Jr., J. M. e Tarbouriech, S. (1999a). Invariance and contractivity of polyhedra for continuous-time linear systems with saturated controls, Revista Controle e Automação da SBA 10(3): 149-158.

Gomes da Silva Jr., J. M. e Tarbouriech, S. (1999b). Polyhedral regions of local asymptotic stability for discretetime linear sytems with saturating controls, IEEETrans. on Automatic Control 44: 2081-2086.

Gomes da Silva Jr., J. M. e Tarbouriech, S. (1999c). Stability regions for linear systems with saturating controls, Proc. of the European Control Conference (ECC'99), Karlsrhue, Germany.

Gomes da Silva Jr, J. M. e Tarbouriech, S. (2001). Local stabilization of discrete-time linear systems with saturating controls: an LMI-based approach, IEEE-Trans. on Automatic Control 46: 119-125.

Henrion, D. e Tarbouriech, S. (1999). LMI relaxations for robust stability of linear systems with saturating controls, Automatica 35: 1599-1604.
Hindi, H. e Boyd, S. (1998). Analysis of linear systems with saturation using convex optimization, Proc. of 37th IEEE Conference on Decision and Control (CDC'98), Tampa, USA, pp. 903-908.

Hu, T. e Lin, Z. (2000). An analysis and design method for linear systems subject to actuator saturation and disturbance, Proc. of the American Control Conference (ACC'O0), Chicago, USA.

Johansson, M. e Rantzer, A. (1998). Computation of piecewise quadratic lyapunov functions for hybrid systems, IEEE-Trans. on Automatic Control 43(4): 555-559.

Khalil, H. K. (1992). Nonlinear Systems, MacMillan.

Molchanov, A. e Pyatnitskii, E. (1989). Criteria of asymptotic stability of differential and difference inclusions encountered in control theory, Systems \& Control Letters 13: 59-64.

Pittet, C., Tarbouriech, S. e Burgat, C. (1997). Stability regions for linear systems with saturating controls via circle and popov criteria, Proc. of 36th IEEE Conference on Decision and Control (CDC'97), San Diego, USA, pp. 4518-4523.

Sussmann, H., Sontag, E. e Yang, Y. (1994). A general result on the stabilization of linear systems using bounded controls, IEEE-Trans. on Automatic Control 39(12): 2411-2425. 